\title{
Factores relacionados con el riesyo de reintervención en pacientes operados de reducción cerradla de fractura de huesos propios
}

\section{Factors related to the risk of reoperation in patients operated on for closed reduction of nasal bone fractures}

Francisco Javier PACHECO COMPAÑA*, Esther RODRÍGUEZ PÉREZ*

Juan Ignacio BUGALLO SANZ**, Alejandro FERNÁNDEZ QUINTO**, Alba GONZÁLEZ RODRÍGUEZ*

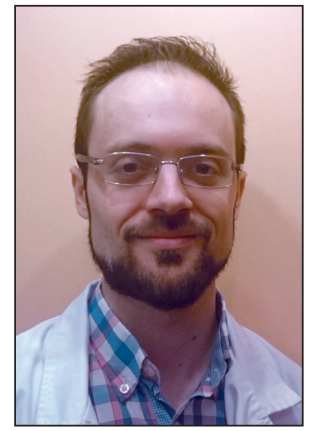

Pacheco Compaña F.J.

\section{Resumen}

Introducción y objetivo. La fractura de huesos nasales es la fractura facial más frecuente. En relación a la cirugía, la técnica habitualmente empleada ante ella es la reducción cerrada bajo anestesia local o general.

Presentamos nuestra experiencia en pacientes intervenidos de fractura de huesos nasales y valoramos el riesgo de reintervención en relación al tipo de técnica anestésica empleada.

Material y método. Recopilamos datos de manera retrospectiva de pacientes intervenidos de reducción cerrada de fractura nasal en nuestro centro entre diciembre de 2014 y junio de 2019. Estudiamos la variable cualitativa analizada (la reintervención) con el test exacto de Fisher y las variables cuantitativas analizadas (días desde el traumatismo hasta cirugía y grados de desviación nasal) con el test U de Mann-Whitney.

Resultados. Incluimos 128 pacientes (91 hombres y 37 mujeres). La causa de fractura más frecuente fue la agresión, seguida por el traumatismo por caída y los deportes. Las fracturas por agresión se produjeron con mayor frecuencia los sábados y domingos entre las 3 y las 5 horas de la madrugada. Las fracturas por caída los lunes y martes a la 1 del mediodía. En 99 casos la cirugía se llevó a cabo en las primeras 24 horas, y en 29 pasado ese tiempo. Con respecto a la técnica anestésica, 9 pacientes fueron intervenidos bajo anestesia local, 24 bajo sedación y 95 bajo anestesia general. Tres de los intervenidos con anestesia local y 4 con anestesia general precisaron reintervención por mal resultado tras la primera cirugía. Ninguno de los pacientes intervenidos con sedación fue reintervenido.

Conclusiones. En nuestra experiencia, la reducción de la fractura nasal bajo sedación tiene buenos resultados, por lo que consideramos que es una buena alternativa en los casos de fractura nasal quirúrgica para reducir los efectos adversos de la anestesia general.

\begin{tabular}{|lr|}
\hline Palabras clave & $\begin{array}{l}\text { Huesos nasales, Fractura huesos nasales, } \\
\text { Reducción cerrada, Anestesia. }\end{array}$ \\
& \\
Nivel de evidencia científica & $4 c$ Terapéutico \\
Recihido [esta versión] & 25 julio/2020 \\
Aceptado & 20 enero/2021
\end{tabular}

Background and objective. Nasal bone fracture is the most frequent facial fracture. In relation to surgery, the most frequently employed technique is closed reduction under local or under general anesthesia.

We present our experience with patients who underwent nasal bone fracture surgery and the risk of reintervention in relation to the type of anesthetic technique used.

Methods. Data were collected retrospectively of patients who underwent closed reduction of nasal fracture in our center between December 2014 and June 2019. Qualitative variable analyzed (reintervention) were studied with Fisher's exact test and the quantitative variables analyzed (days from trauma to surgery and degrees of nasal deviation) with the Mann-Whitney U test.

Results. One hundred and twenty-eight patients (91 men and 37 women) were included in the study. The most frequent cause of fracture was aggression, followed by falls and sports. Aggression fractures occurred most frequently on Saturdays and Sundays between 3 and 5 a.m. Fall fractures occurred most frequently on Mondays and Tuesdays at 1 p.m. In 99 cases the surgery was performed in the first 24 hours, while in 29 cases it was performed after that time. Regarding the anesthetic technique, 9 patients were operated under local anesthesia, 24 under sedation and 95 under general anesthesia. Three of the patients operated with local anesthesia and 4 with general anesthesia required reoperation because of poor results after the first surgery. None of the patients operated with sedation were reoperated.

Conclusions. In our experience, nasal fracture reduction under sedation has good results making it a good alternative in cases of surgical nasal fracture reducing the deleterious effects of general anesthesia.

$\begin{array}{lr}\text { Key words } & \begin{array}{l}\text { Nasal bones, Nasal bones fracture, } \\ \text { Close reduction, Anesthesia. }\end{array} \\ \begin{array}{l}\text { Level of evidence } \\ \text { Received [this version] } \\ \text { Accepted }\end{array} & 4 c \text { Therapeutic } \\ \end{array}$

Conflicto de intereses: Los autores declaran no tener ningún interés financiero relacionado con el contenido de este artículo. Financiación: No hubo fuentes externas de financiación para este trabajo. 
Introducción

Los traumatismos faciales son uno de los motivos más frecuentes de consulta en los Servicios de Urgencias. Dentro de éstos, la fractura de huesos propios (o nasales) se considera la fractura facial más frecuente y supone una importante carga de trabajo en centros de atención primaria y hospitales. Sin embargo, desde el punto de vista quirúrgico, la mayoría de los pacientes con diagnóstico de fractura nasal no son intervenidos quirúrgicamente por tratarse de fracturas no desplazadas o por ser pacientes con poca exigencia estética y/o funcional (como pacientes muy añosos o pluripatológicos).

En relación a la cirugía, la técnica habitualmente empleada ante una fractura de huesos nasales es la reducción cerrada, que puede llevarse a cabo bajo anestesia local o general. Varias publicaciones afirman que los paciente intervenidos de reducción cerrada de huesos nasales bajo anestesia general presentan mayor satisfacción con los resultados obtenidos, a la par que disminuye el número de reintervenciones. ${ }^{(1,2)}$ Otras publicaciones sin embargo, defienden que los resultados obtenidos tras la reducción con anestesia local son similares a los obtenidos bajo anestesia general. ${ }^{(3-5)}$

El objetivo del presente estudio es realizar un análisis descriptivo de nuestra experiencia en pacientes intervenidos de reducción cerrada de fractura nasal, analizando sus principales causas y valorando el tipo de anestesia empleada y el número de reintervenciones que fueron precisas.

Material y método

Recopilamos de forma retrospectiva datos de pacientes intervenidos por fractura nasal en el Servicio de Cirugía Plástica del Complejo Hospitalario Universitario de A Coruña (España), entre diciembre de 2014 y junio de 2019. Los criterios de inclusión para la recogida de pacientes fueron los siguientes: pacientes intervenidos de fractura nasal, cirugía cerrada de reducción de huesos nasales. Como criterios de exclusión determinamos: pacientes menores de 16 años de edad, si precisaron de reducción abierta de la fractura en algún momento, si fue preciso realizar septoplastia asociada o si se perdió el seguimiento posoperatorio (el paciente no acudió a consulta).

Las variables estudiadas fueron: edad, sexo, fecha y hora de la fractura; causa de la misma; pruebas de imagen; fecha de la cirugía; tipo de anestesia y reintervención. Otras variables accesorias estudiadas fueron: taponamiento empleado; pomada que impregna el taponamiento; ferulización; retirada del taponamiento y de la férula.
Para el análisis estadístico empleamos el programa SPSS versión 21.0. Estudiamos la variable cualitativa analizada (la reintervención) con el test exacto de Fisher y las variables cuantitativas analizadas (días desde el traumatismo hasta la cirugía y grados de desviación nasal) con el test U de Mann-Whitney.

\section{Técnica quirúrgica y anestésica}

La reducción cerrada de huesos nasales puede llevarse a cabo de manera manual cuando existen resaltes óseos que precisan reducción mediante compresión externa. Para elevar las fracturas hundidas, sin embargo, es preciso emplear un fórceps de Walsham o un mango de bisturí introducido a través de la narina. Mediante uno de estos dos elementos elevamos el hueso hundido. Posteriormente procedemos al taponamiento nasal anterior y la colocación de una férula de yeso en el dorso nasal.

Para la anestesia local utilizamos infiltración local con mepivacaína al $2 \%$ en la base del septo nasal y en el dorso de la raíz nasal, a la que sumamos adrenalina 1: 100.000 para reducir el riesgo de sangrado. Empleamos además lidocaína pulverizada (10 mg/pulsación) a través de ambas fosas nasales. En pacientes en los que la anestesia fue general, realizamos 2 técnicas diferentes: anestesia general con intubación orotraqueal o sedación sin intubación; esta última puede acompañarse de infiltración local de anestesia.

\section{Resultados}

En total, incluimos en el estudio 128 pacientes, de los cuales 91 fueron hombres y 37 mujeres (ratio 2.46). La edad media fue de 35.36 años (32.86 en hombres y 41.51 en mujeres), con un rango entre 16.10 y 84.76 años.

La causa de fractura nasal más frecuente fue la agresión $(43 \% ; n=55)$ seguida por el traumatismo por caída $(25 \% ; n=32)$, deportes $(10 \% ; n=13)$, accidentes de tráfico $(6 \% ; n=8)$, traumatismos contra una puerta o pared $(4 \% ; n=5)$ y traumatismos con objetos $(3 \% ; n=4)$. En 11 casos $(8.5 \%)$ la fractura se produjo por otra causa o la causa era desconocida. En relación al género, la causa más frecuente entre los hombres fue la agresión (49\% de las fracturas en hombres; $n=45$ ) mientras que en las mujeres fue la caída (51\% de las fracturas en mujeres; $\mathrm{n}=19)$. La edad media de los pacientes que sufrieron agresión fue de 28.85 años (máxima de 63.63 y mínima de 17.30 años) y la edad media en los pacientes que sufrieron caídas fue de 49.96 años (máxima de 84.76 y mínima de 16.10). El deporte en el que se produjeron más fracturas nasales quirúrgicas fue el fútbol, seguido del ciclismo (Tabla I). Sólo en 15 casos quedó reflejado en la historia clínica el consumo de alcohol por parte del 
Tabla I. Número de pacientes en relación a la causa de la fractura de huesos nasales

\begin{tabular}{|c|c|c|c|}
\hline \multicolumn{2}{|c|}{ Causa de la fractura } & $\mathbf{N}$ & $\%$ \\
\hline \multicolumn{2}{|l|}{ Agresión } & 55 & 43 \\
\hline \multicolumn{2}{|l|}{ Caída } & 32 & 25 \\
\hline \multirow{7}{*}{ Deporte } & Fútbol & 4 & 3 \\
\hline & Bicicleta & 3 & 2 \\
\hline & Surf & 2 & 1 \\
\hline & Baloncesto & 1 & 0.7 \\
\hline & Béisbol & 1 & 0.7 \\
\hline & Esquí & 1 & 0.7 \\
\hline & Pesas & 1 & 0.7 \\
\hline \multicolumn{2}{|l|}{ Tráfico } & 8 & 6 \\
\hline \multicolumn{2}{|c|}{ Trauma con puerta/pared } & 5 & 4 \\
\hline \multicolumn{2}{|c|}{ Trauma con objeto } & 4 & 3 \\
\hline \multicolumn{2}{|c|}{ Otros/desconocido } & 11 & 13 \\
\hline
\end{tabular}

paciente a su llegada a urgencias, la mayoría de los cuales habían sufrido agresión (13 de los 15 casos).

La prueba de imagen realizada con mayor frecuencia fue la radiografía de huesos propios nasales $(62 \%$; $\mathrm{n}=79$ ). Se realizó una tomografía computerizada (TC) en 40 pacientes $(31 \%)$ y en 6 pacientes $(5 \%)$ se realizaron ambas pruebas. En 10 casos (7.8\%) el paciente presentaba otras fracturas faciales asociadas, 2 de los cuales habían sido intervenidos bajo anestesia local y 8 en presencia de anestesista $(\mathrm{p}=0.147)$. En los pacientes en los que se realizó una TC se midió el ángulo de desviación que presentaba la fractura nasal (en su punto de mayor angulación). La media general de desviación fue de $22.2^{\circ}$ (máximo de $50^{\circ}$ y mínimo de $4^{\circ}$ ).

En relación con la hora del día, las fracturas por agresión se produjeron con mayor frecuencia entre las 3 y las 5 horas de la madrugada. La hora más frecuente para las fracturas por caída fue la 1 del mediodía (Fig. 1). Con respecto a los días de la semana, las fracturas por agresión fueron más frecuentes en sábados y domingos mientras que las caídas lo fueron en lunes y martes (Fig. 2). A lo largo del año las fracturas por agresiones presentan altibajos, si bien las caídas son mucho más frecuentes en diciembre (Fig. 3).

En 99 casos (77.3\%) la cirugía se llevó a cabo en las primeras 24 horas, mientras que en 29 casos $(22.6 \%)$ se

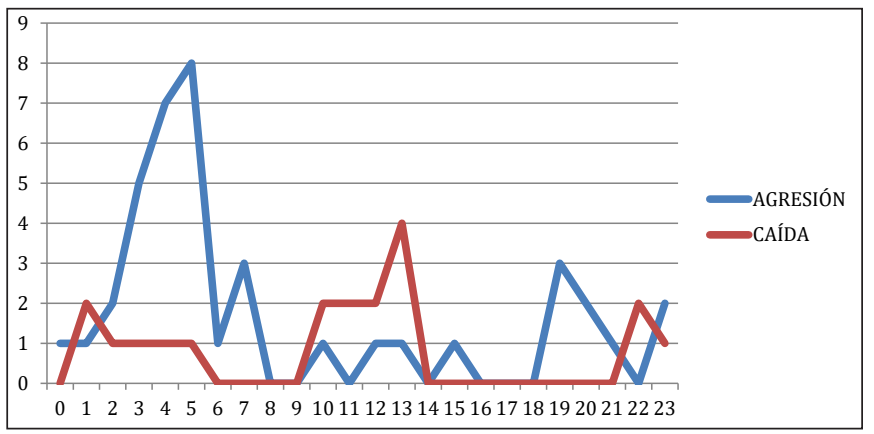

Figura 1. Número de pacientes en relación a la hora del día en que se produjo el traumatismo en función de la causa del traumatismo.

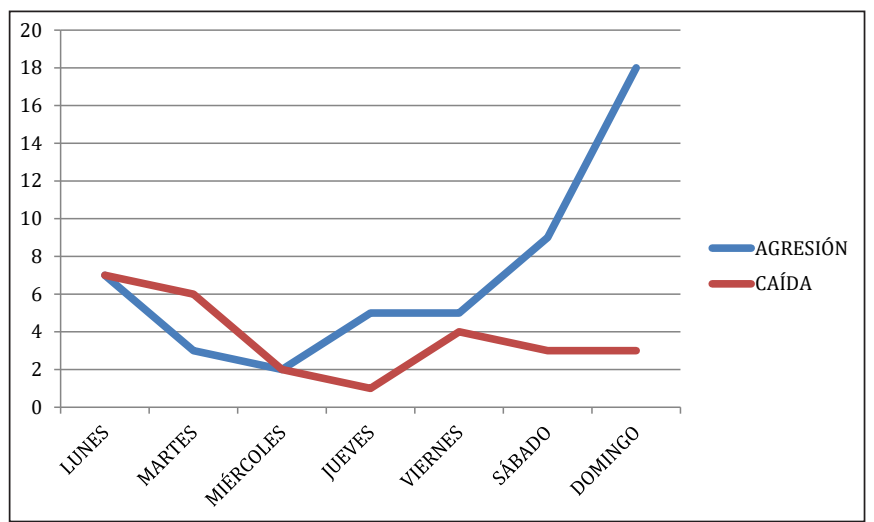

Figura 2. Número de pacientes en relación al día de la semana en que se produjo el traumatismo en función de la causa del traumatismo.

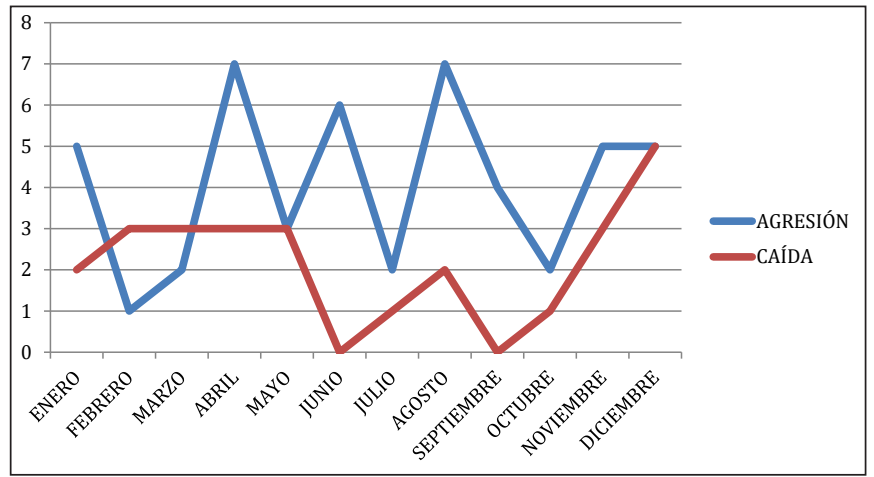

Figura 3. Número de pacientes en relación al mes del año en que se produjo el traumatismo en función de la causa del traumatismo.

realizó pasado ese tiempo. La media de días en pacientes intervenidos pasadas las 24 horas fue de 12.31 días (máximo de 37 días y mínimo de 2) (Tabla II).

Recogimos también información sobre el material empleado para el taponamiento nasal en 60 casos, siendo el más empleado el taponamiento con gasa de bordes orillada (48 casos). En 50 casos registramos además la pomada empleada para impregnar el material de tapona-

Tabla II. Análisis de variables cuantitativas (días hasta la cirugía, de retirada del taponamiento nasal y de retirada de la férula nasal)

\begin{tabular}{|c|c|c|c|c|c|c|c|}
\hline Variable & $\mathbf{N}$ & Media & Mediana & Mínimo & Máximo & DT & $p$ \\
\hline Días hasta la cirugía & 128 & 2.99 & 0 & 0 & 37 & 6.73 & \\
\hline - No reintervenidos & 121 & 3.12 & 0 & 0 & 37 & 6.87 & \multirow{2}{*}{0.154} \\
\hline - Reintervenidos & 7 & 0.86 & 0 & 0 & 6 & 2.27 & \\
\hline Días hasta la cirugía (más de 24 horas) & 29 & 12.31 & 10 & 2 & 37 & 9.40 & \\
\hline Retirada taponamiento & 88 & 3.97 & 4 & 1 & 9 & 1.56 & \\
\hline Retirada férula & 91 & 9.89 & 10 & 3 & 17 & 2.83 & \\
\hline
\end{tabular}


Tabla III. Materiales empleados para el taponamiento y pomadas empleadas para impregnar dichos materiales

\begin{tabular}{|l|c|c|}
\hline & N & \% \\
\hline TAPONAMIENTO & 60 & 100 \\
\hline Gasa de bordes orillada & 48 & 80 \\
\hline Merocel $^{\circledR *}$ & 12 & 20 \\
\hline POMADA & 49 & 100 \\
\hline Nitrofural 2mg/g & 35 & 71.43 \\
\hline Mupirocina 20mg/g & 12 & 24.49 \\
\hline Eritromicina $5 \mathrm{mg} / \mathrm{g}$ & 1 & 2.04 \\
\hline Vaselina & 1 & 2.04 \\
\hline
\end{tabular}

* Merocel $^{\circledR}$ (Medtronic Xomed Inc; Jacksonville, EEUU).

Tabla IV. Número de pacientes en relación a la técnica anestésica empleada y su relación con la reintervención

\begin{tabular}{|l|c|c|c|c|}
\hline \multirow{2}{*}{ Técnica anestésica } & \multicolumn{2}{|c|}{ Total } & \multicolumn{2}{c|}{ Reintervención } \\
\cline { 2 - 5 } & $\mathrm{N}$ & $\%$ & $\mathrm{~N}$ & $\%$ \\
\hline Local & 9 & 7 & 3 & 2 \\
\hline Sedación & 24 & 18.8 & 0 & 0 \\
\hline General & 95 & 74.2 & 4 & 3 \\
\hline
\end{tabular}

Tabla V. Valores de significación estadística al relacionar la técnica anestésica en función del número de pacientes que precisaron reintervención

\begin{tabular}{|l|c|}
\hline Técnica anestésica & $\mathbf{p}$ \\
\hline Local-General/Sedación & 0.007 \\
\hline Local-General & 0.013 \\
\hline Local-Sedación & 0.015 \\
\hline Sedación-General & 0.582 \\
\hline
\end{tabular}

Tabla Vl: Valores de los ángulos de desviación de la fractura nasal medidos en la TC.

\begin{tabular}{|c|c|c|c|c|c|}
\hline Anestesia & Media & Mediana & N & Desv. típ. & p* \\
\hline General & 19,767 & 18,000 & 30 & 11,4545 & 0,005 \\
\hline Local & 21,000 & 20,000 & 5 & 9,0830 & 0,016 \\
\hline Sedación & 38,000 & 34,000 & 5 & 8,2765 & \\
\hline Total & 22,200 & 19,500 & 40 & 12,4821 & \\
\hline
\end{tabular}

* La p de significación estadística relaciona cada anestesia frente a la sedación.

miento, siendo la más frecuente la pomada de nitrofural $2 \mathrm{mg} / \mathrm{g}$ (35 casos) (Tabla III). En los pacientes en los que se había colocado taponamiento nasal (107 pacientes), el tiempo medio de retirada de los mismos fue de 3.97 días (máximo de 9 días y mínimo de 1). En cuanto al tiempo medio de retirada de la férula nasal, dato que registramos en 91 pacientes, fue 9.89 días (Tabla II).

Con respecto a la técnica anestésica empleada, 9 pacientes $(7 \%)$ fueron intervenidos bajo anestesia local y $119(93 \%)$ en presencia de un anestesista, de los cuales 24 pacientes $(19 \%)$ fueron sometidos a sedación y 95 (74\%) bajo anestesia general con intubación orotraqueal.

Del total de pacientes operados fueron reintervenidos $7(5.5 \%)$ por una reducción incompleta de la fractura. La media de días entre el traumatismo y la intervención en pacientes operados con anestesia local fue de 0.11 días, mientras que la media de días en pacientes intervenidos en presencia de anestesista fue de 3.21 días ( 0.33 días en pacientes intervenidos bajo sedación y 3.94 días en pacientes intervenidos bajo anestesia general), siendo dichas diferencias estadísticamente significativas $(\mathrm{p}=0.040)$.

La media de días desde el traumatismo hasta la intervención en los pacientes que no precisaron reintervención fue 3.12 días y en los que pacientes que sí fueron reintervenidos fue de 0.86 días, no siendo estas diferencias estadísticamente significativas $(\mathrm{p}=0.154)$.

Encontramos en nuestro estudio que 3 de los pacientes intervenidos con anestesia local y 4 de los pacientes intervenidos con anestesia general precisaron reintervención por mal resultado tras la primera cirugía. Ninguno de los pacientes intervenidos con sedación fue reintervenido (Tabla IV). Si comparamos el número de pacientes intervenidos con anestesia local frente a los intervenidos con anestesia general o sedación observamos que existen diferencias estadísticamente significativas $(\mathrm{p}=0.007)$, siendo más probable la reintervención con anestesia local. No encontramos diferencias estadísticamente significativas en el número de reintervenciones entre el empleo de anestesia general o la sedación $(\mathrm{p}=0.582)$ (Tabla $\mathrm{V})$.

En relación al ángulo de desviación de los huesos fracturados objetivamos que era similar en pacientes intervenidos con anestesia local y con general $\left(21^{\circ}\right.$ y $19.77^{\circ}$ respectivamente) y mayor ángulo de manera estadísticamente significativa en los pacientes intervenidos con sedación $\left(38^{\circ}\right)$ (Tabla VI). En pacientes que precisaron reintervención la media del ángulo fue de $26^{\circ}$, siendo de $21.89^{\circ}$ en los que no precisaron reintervención, sin encontrar diferencias estadísticamente significativas $(\mathrm{p}=0.557)$.

\section{Discusión}

En nuestra experiencia, encontramos que la incidencia de reintervención tras reducción cerrada de fractura de huesos nasales aumenta en pacientes intervenidos con anestesia local, si bien no hay diferencias entre los pacientes intervenidos con anestesia general o sedación.

Hay que tener en cuenta que el estudio parte de una limitación importante. El escaso número de pacientes intervenidos con anestesia local hace que nuestros resultados deban ser tomados con cautela en ese aspecto. Así mismo otra limitación es que fueron diferentes cirujanos los que llevaron a cabo la infiltración anestésica, pudiendo haber variaciones en la técnica de unos a otros. Sin embargo, consideramos que es interesante poder proponer la sedación como alternativa a la anestesia general en 
pacientes intervenidos para reducción cerrada de fractura de huesos nasales.

En relación a la literatura revisada, varias publicaciones afirman que los paciente intervenidos para reducción cerrada de fractura de huesos nasales bajo anestesia general presentan mayor satisfacción con los resultados obtenidos y se reduce en ellos el número de reintervenciones. ${ }^{(1,2)}$ Otras publicaciones, sin embargo, defienden que los resultados obtenidos tras la reducción con anestesia local son similares a los obtenidos bajo anestesia general. ${ }^{(5,6)}$ En nuestro caso, la necesidad de reintervención se asoció a un mal resultado estético con mala reducción de la fractura. Algunos artículos que refieren que los resultados de la reducción nasal con anestesia local son satisfactorios, no comparan la técnica con la anestesia general. ${ }^{(3,7)}$ Otros autores concluyen que la elección entre anestesia local o anestesia general no modifica la satisfacción del paciente con los resultados y la tasa de fallos de la técnica, si bien añaden que la elección de anestesia local es beneficiosa en casos seleccionados ${ }^{(8)}$ y otros excluyen fracturas conminutas con afectación septal. ${ }^{(9)}$ Otros autores argumentan que aunque la satisfacción es alta en general, los pacientes que son intervenidos con anestesia local suelen requerir con más frecuencia una nueva cirugía. ${ }^{(4)}$

En nuestro estudio, todos los casos en los que la reducción de la fractura de huesos nasales se había hecho con anestesia local y que fueron reintervenidos, habían sido operados en las primeras 24 horas tras la lesión, mientras que 1 de los casos en los que la reducción se hizo bajo anestesia general y precisó reintervención, había sido operado a los 6 días. Ninguno de los casos reintervenidos presentaba otras fracturas faciales asociadas.

En relación al tiempo que trascurre entre el traumatismo y la cirugía existe cierta controversia en la literatura publicada. Por lo general, en aquellos pacientes en los que no es posible llevar a cabo una cirugía urgente se prefiere realizar el procedimiento en las primeras 2 semanas tras el traumatismo. ${ }^{(10-12)}$ Otros autores, sin embargo, publican que hasta aproximadamente los 30 días los resultados son similares a los obtenidos en menos tiempo. ${ }^{(13,6)}$ Algunos incluso refieren que esperar para realizar la reducción al menos 3 días tras el traumatismo reduce de manera significativa el sangrado ${ }^{(3)}$ y facilita la cirugía al reducir el edema postraumático. ${ }^{(14)}$ En nuestro caso, la mayoría de los pacientes fueron intervenidos en las primeras 24 horas tras la lesión. No encontramos diferencias estadísticamente significativas en el tiempo de demora hasta la cirugía entre los que precisaron reintervención y los que no.

En relación al taponamiento nasal, las publicaciones revisadas sugieren que el tiempo de mantenimiento del mismo sea de entre 3-5 días. ${ }^{(15,16)}$ La férula en dorso na- sal habitualmente se mantiene una semana. ${ }^{(11,14,16,17)}$ Estos datos son similares a los obtenidos en nuestro estudio.

Un artículo publicado en Estados Unidos en 2019, declaran que la causa más frecuente de las fracturas faciales en pacientes jóvenes eran los deportes, ${ }^{(18)}$ siendo el de mayor frecuencia el baloncesto, así como el béisbol, fútbol americano, sóftbol, ciclismo y soccer (fútbol europeo). ${ }^{(17,18)}$ Otro estudio publicado en Corea del Sur en 2012 refiere que la primera causa de fractura nasal son los deportes, seguidos de los accidentes de tráfico y las caídas. ${ }^{(16)}$ Por otro lado, otro estudio hecho en Corea del Sur en 2014 señala que las causas más frecuentes fueron las caídas. ${ }^{(12)}$ En nuestro estudio, la causa más frecuente fue la agresión y esto es debido a que solo incluyó pacientes quirúrgicos, quedando fuera pacientes muy añosos que no fueron intervenidos y en los que la causa de fractura nasal fue una caída. Este hallazgo concuerda con otros estudios en los que solo se contabilizaron pacientes intervenidos quirúrgicamente. ${ }^{(3,11)}$ Entre los deportes, el que con mayor frecuencia se asoció a fracturas nasales quirúrgicas en nuestro estudio fue el fútbol. Hay que tener en cuenta que este es el deporte mayoritariamente practicado en España, por lo que también es lógico que sea el que mayor número de fracturas produce. En relación a la hora en que se producen las fracturas, la literatura concuerda con nuestros resultados en que la mayoría son entre las 12 de la noche y las 7 de la mañana, ${ }^{(19)} y$ con mayor frecuencia durante el fin de semana. ${ }^{(3)}$

\section{Conclusiones}

La fractura de huesos nasales es una consulta muy frecuente en los servicios de urgencias hospitalarias. En nuestra experiencia y en base al estudio de recogida y análisis de casos realizado, consideramos que la reducción cerrada de esta fractura en las primeras horas y el uso de anestesia bajo sedación ha proporcionado buenos resultados.

Consideramos por tanto, que esta técnica anestésica debería ser valorada de forma positiva en casos de fractura nasal que precise reducción quirúrgica para evitar así o disminuir los efectos adversos de la anestesia general.

\section{Dirección del autor}

\section{Dr. Francisco Javier Pacheco Compaña}

Servicio de Cirugía Plástica

Complejo Hospitalario de A Coruña

Avda. de Ax Xubias 84

15006, A Coruña, España

Correo electrónico: javipacheco25@hotmail.com 


\section{Bibliografía}

1. Al-Moraissi EA, Ellis E. Local versus general anesthesia for the management of nasal bone fractures: a systematic review and meta-analysis. J Oral Maxillofac Surg. 2015;73(4):606-615.

2. Courtney MJ, Rajapakse Y, Duncan G, Morrissey G. Nasal fracture manipulation: a comparative study of general and local anaesthesia techniques. Clin Otolaryngol Allied Sci. 2003;28(5):472-475.

3. Vilela F, Granjeiro R, Maurício C, Andrade P. Applicability and Effectiveness of Closed Reduction of Nasal Fractures under Local Anesthesia. Int Arch Otorhinolaryngol. 2014;18(3):266271.

4. Rajapakse Y, Courtney M, Bialostocki A, Duncan G, Morrissey G. Nasal fractures: a study comparing local and general anaesthesia techniques. ANZ J Surg. 2003;73(6):396-399.

5. Khwaja S, Pahade AV, Luff D, Green MW, Green KMJ. Nasal fracture reduction: local versus general anaesthesia. Rhinology. 2007;45(1):83-88.

6. Hwang K, Yeom SH, Hwang SH. Complications of Nasal Bone Fractures. J Craniofac Surg. 2017;28(3):803-805.

7. Pérez-García A, Esteban-Vico JR, Lorca-García C, García-Sánchez JM, Miranda Gómez $\mathrm{L}$, Llinás Porte A, et al. Endonasal Infiltrative Anesthesia for Nasal Fracture Reduction. Plast Surg Nurs. 2019;39(1):22-24.

8. Atighechi S, Baradaranfar MH, Akbari SA. Reduction of nasal bone fractures: a comparative study of general, local, and topical anesthesia techniques. J Craniofac Surg. 2009;20(2):382384.

9. Lee Y-J, Lee K-T, Pyon J-K. Finger reduction of nasal bone fracture under local anesthesia: outcomes and patient reported satisfaction. Arch Craniofac Surg. 2019;20(1):24-30.
10. James JG, Izam AS, Nabil S, Rahman NA, Ramli R. Closed and Open Reduction of Nasal Fractures. J Craniofac Surg. 2020;31(1):22-26.

11. Sharma SD, Kwame I, Almeyda J. Patient aesthetic satisfaction with timing of nasal fracture manipulation. Surg Res Pract. 2014;2014:238520.

12. Park H-K, Lee J-Y, Song J-M, Kim T-S, Shin S-H. The Retrospective Study of Closed Reduction of Nasal Bone Fracture. Maxillofac Plast Reconstr Surg. 2014;36(6):266-272.

13. Perkins V, Vijendren A, Egan M, McRae D. Optimal timing for nasal fracture manipulation-Is a 2-week target really necessary? A single-centre retrospective analysis of 50 patients. Clinical Otolaryngology. 2017;42(6):1377-1381.

14. Yilmaz MS, Guven M, Varli AF. Nasal fractures: is closed reduction satisfying? J Craniofac Surg. 2013;24(1):e36-38.

15. Jang N, Shin HW. Are postoperative prophylactic antibiotics in closed reduction of nasal bone fracture valuable?: prospective study of 30 cases. Arch Craniofac Surg. 2019;20(2):89-93.

16. Kim JH, Lee JH, Hong SM, Park CH. Open reduction of nasal bone fractures through an intercartilaginous incision. Acta Otolaryngol. 2013;133(1):77-81.

17. Marston AP, O'Brien EK, Hamilton GS. Nasal Injuries in Sports. Clin Sports Med. 2017;36(2):337-53.

18. Povolotskiy R, Youssef P, Kaye R, Paskhover B. Facial Fractures in Young Adults: A National Retrospective Study. Ann Otol Rhinol Laryngol. 2019;128(6):516-523.

19. Kuhr E, Werner JA, Dietz A, Wiegand S. Analysis of the emergency patients of a university ENT hospital. Laryngorhinootologie. 2019;98(9):625-630. 